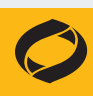

Promega

\title{
Faster, cleaner bisulfite conversion that yields more intact DNA for downstream analysis
}

\author{
Performing DNA purification, bisulfite conversion and amplification are crucial steps in DNA \\ methylation analysis, especially when working with difficult sample types like formalin-fixed paraffin- \\ embedded (FFPE) tissue. The MethylEdge ${ }^{\mathrm{TM}}$ Bisulfite Conversion System provides a rapid, efficient \\ method for bisulfite conversion with minimal DNA fragmentation in less than $2 \mathrm{~h}$. Coupled with \\ purification chemistries optimized for FFPE tissue and flexible, robust amplification technologies for \\ detection, the MethylEdge ${ }^{\mathrm{TM}}$ Bisulfite Conversion System delivers high-quality converted DNA for \\ downstream analysis.
}

\section{Introduction}

Methylation of cytosines in $\mathrm{CpG}$ dinucleotides is an epigenetic mechanism that contributes to regulation of gene expression. Bisulfite conversion is an essential tool for studying DNA methylation patterns. Unmethylated cytosines react with bisulfite and are converted to uracils, whereas methylated cytosines are unaffected. This allows sequence-specific determination of cytosine methylation, which is of interest to researchers studying regulation of gene expression in normal and diseased tissue samples. The MethylEdge ${ }^{T M}$ Bisulfite Conversion System from Promega provides chemistry to perform this reaction using DNA from a variety of sample types. Tissue samples are often FFPE for archiving purposes. Nucleic acids can be extracted from FFPE samples for use in PCR and sequencing applications, but extraction procedures can result in highly fragmented DNA due to degradation from harsh fixation procedures and long-term storage. Analyzing the methylation status of DNA from FFPE tissue samples can be challenging because the typically harsh conditions of bisulfite conversion (incubation at low $\mathrm{pH}$ and high temperature) can result in further fragmentation and sample loss, leaving a small amount of highly fragmented DNA, which may not be suitable for downstream applications. Promega DNA extraction systems for FFPE tissue, available in manual and automated formats, do not require hazardous reagents or overnight incubation and are optimized to yield high-quality, amplifiable DNA. The MethylEdge ${ }^{\mathrm{TM}}$ Bisulfite Conversion System limits DNA fragmentation while maintaining conversion efficiency.

We evaluated bisulfite conversion of DNA extracted from FFPE tissue samples by comparing the MethylEdge ${ }^{\mathrm{TM}}$ Bisulfite Conversion System with two other commercially available bisulfite conversion kits.

Jennifer Mook, Amy Hendricksen \& Karen Reece

Promega, Madison, Wisconsin, USA. Correspondence should be addressed to A.H. (amy.hendricksen@promega.com).

\section{MethylEdge ${ }^{\mathrm{TM}}$ methodology}

DNA was extracted from two $5 \mu \mathrm{m}$ human liver FFPE sections using either ReliaPrep ${ }^{\text {TM }}$ FFPE gDNA Miniprep System or the automated Maxwell ${ }^{\circledR} 16$ FFPE Tissue LEV DNA Purification Kit following the manufacturer's instructions. Eluted DNA for each kit was pooled, and DNA concentration was determined using spectrophotometry and adjusted to $17.5 \mathrm{ng} / \mu \mathrm{L}$. The purified DNA (350 ng) underwent bisulfite conversion using either the MethylEdge ${ }^{\mathrm{TM}}$ Bisulfite Conversion System, kit A or kit B according to the manufacturers' instructions ( $n=3$ for each kit). Following bisulfite conversion, DNA concentration was estimated using spectrophotometry by measuring absorbance at 220$350 \mathrm{~nm}$ to determine percent recovery ((eluted DNA/input DNA) $\times$ 100). We used $20 \mathrm{ng}$ from each reaction as template DNA in a duplex, bisulfite-specific real-time PCR ( $n=3$ for each conversion reaction) using Promega GoTaq ${ }^{\circledR}$ qPCR Master Mix with one primer set specific for converted DNA and another specific for unconverted DNA.

\section{MethylEdge ${ }^{\text {TM }}$ System performance}

DNA concentration following FFPE extraction and purification was $58.7 \mathrm{ng} / \mu \mathrm{L}$ for ReliaPrep ${ }^{\text {TM }}$ FFPE and $24.8 \mathrm{ng} / \mu \mathrm{L}$ for Maxwell ${ }^{\circledR}$ FFPE. Bisulfite conversion reagents were prepared for kit $\mathrm{A}$ and kit $\mathrm{B}$ following the manufacturers' instructions. The MethylEdge ${ }^{\mathrm{TM}}$ reagents were provided ready to use and did not require any upfront preparation. The total time required for bisulfite conversion, desulphonation and cleanup was $1.75 \mathrm{~h}$ for the MethylEdge ${ }^{T M}$ System, $3.5 \mathrm{~h}$ for kit $\mathrm{A}$ and $5 \mathrm{~h}$ for kit B. Percent recovery (Fig. 1) was similar for the MethylEdge ${ }^{\text {TM }}$ System and kit A (average of $60 \%$ with DNA purified using ReliaPrep ${ }^{\mathrm{TM}}$ FFPE kit or Maxwell ${ }^{\circledR} 16$ FFPE). For kit B, recovery was highly variable and 20-40\% lower than that for the MethylEdge ${ }^{\top M}$ System. The absorbance scan of bisulfite-converted DNA with kit B revealed high peaks at $230 \mathrm{~nm}$ (data not shown), which were not seen with DNA converted with either the MethylEdge ${ }^{\mathrm{TM}}$ System or kit A. We recommend 


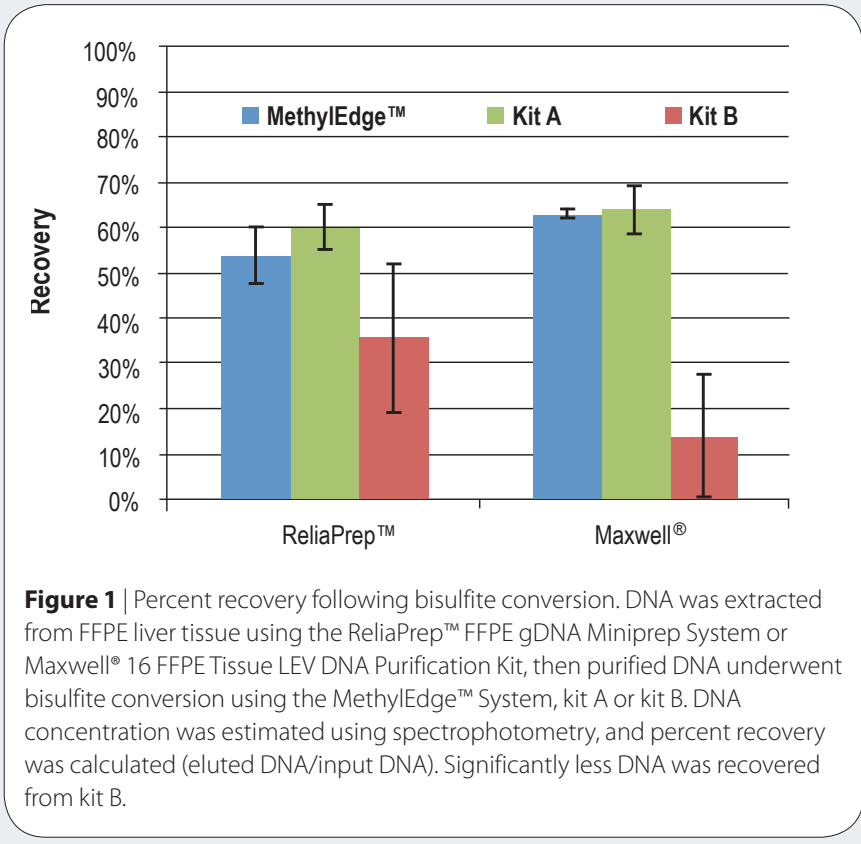

performing an absorbance scan following bisulfite conversion, if possible, to assess quality issues with eluted DNA that may be revealed through measurements at other wavelengths (for example, at 230 and $280 \mathrm{~nm})$.

Bisulfite-converted DNA was analyzed using duplex, bisulfitespecific real-time PCR performed using primers specific for bisulfite-converted DNA (248 bp amplicon) and primers specific for unconverted DNA (310 bp amplicon) to assess performance and conversion efficiency. Experiments included reactions performed with prequalified, unconverted control DNA (Human Genomic DNA, Promega) and bisulfite-converted control DNA (Converted Human Methylated Control, Promega). This PCR assay uses the GoTaq ${ }^{\circledR}$ qPCR Master Mix and assesses conversion efficiency by comparing the melting temperature $\left(T_{m}\right)$ for experimental samples to that of control reactions (Fig. 2). Quantification cycles $\left(C_{q}\right)$ for DNA converted with kit $B$ were significantly later and highly variable. Melting temperatures for two samples converted with kit B also were shifted, indicating possible incomplete conversion. Furthermore, the maximum fluorescent signals for samples converted with kit B were significantly lower than those for all other samples run in this experiment.

\section{Conclusions}

Promega offers automated and manual options for extracting DNA from FFPE tissue without hazardous, organic reagents or overnight incubation. Both protocols are streamlined and yield high-quality DNA suitable for bisulfite conversion experiments. Although DNA converted with the MethylEdge ${ }^{T M}$ Bisulfite Conversion System or kit A performed similarly in real-time PCR, the MethylEdge ${ }^{\text {TM }}$ Bisulfite Conversion System protocol took less time and the room temperature-stable reagents were provided ready to use, minimizing the possibility for error and contamination. GoTaq ${ }^{\circledR}$ qPCR Master Mix efficiently amplified bisulfite-converted DNA (even poor-quality DNA converted with
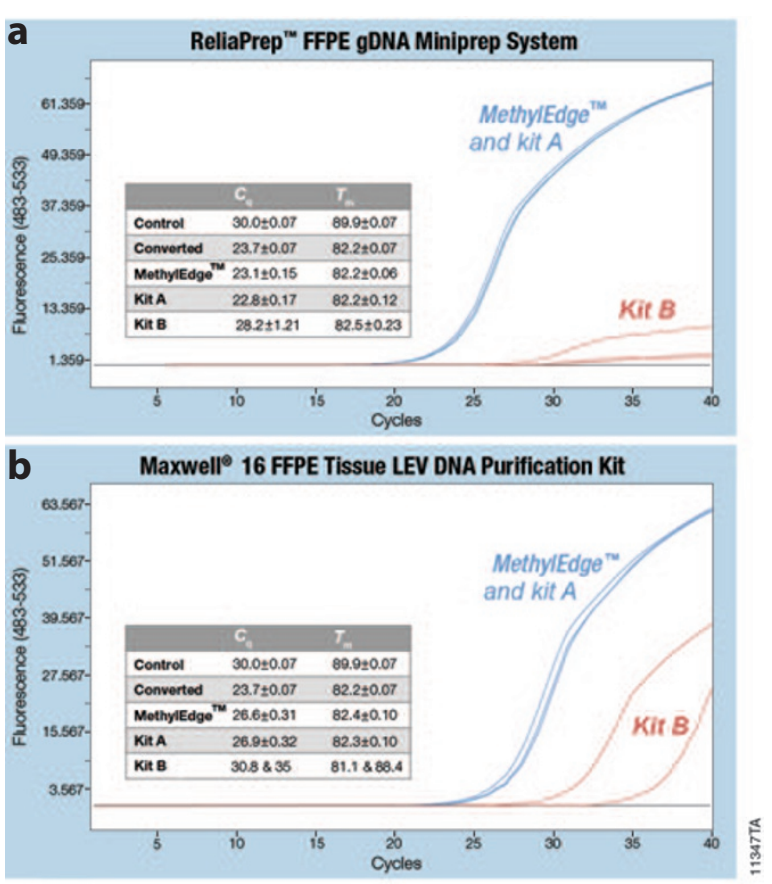

Figure $\mathbf{2}$ | Comparison of real-time PCR results after bisulfite conversion. (a,b) Bisulfite-converted DNA (20 ng) was used as a template in a duplex, real-time PCR capable of detecting both converted and unconverted DNA. Prequalified, unconverted DNA (control) and bisulfite-converted control DNA (converted) samples were run in parallel with experimental samples as controls to verify conversion efficiency and DNA quality. The MethylEdge ${ }^{\text {TM }}$ System and kit A samples (blue) showed robust amplification with similar quantification cycle $\left(C_{q}\right)$ and melting temperature $\left(T_{m}\right)$ values. Kit B samples (red) showed poor amplification. Two kit B samples showed shifted $T_{m}$ values, suggesting incomplete conversion.

kit B) without the need for specialized enzymes or buffer formulations. Promega offers a suite of products that complement a DNA methylation workflow from DNA purification to PCR analysis applications (Fig. 3).

DNA Methylation workflow gld
Purify \&
Bisulfite
Downstream
Quantitate Conversion Analysis

Figure $\mathbf{3}$ | Promega DNA Methylation workflow.

This article was submitted to Nature Methods by a commercial organization and has not been peer reviewed. Nature Methods takes no responsibility for the accuracy or otherwise of the information provided. 